\title{
Kepler's equation and limit cycles in a class of PWM feedback control systems
}

\author{
Alfredo Eisinberg • Giuseppe Fedele · \\ Domenico Frascino
}

Received: date / Accepted: date

\begin{abstract}
The aim of this paper is to point out some new results concerning the ripple instability in the closed loop control system using pulse width modulators (PWM), with natural sampling, as power amplifier. The presented analysis, based on dualinput describing function method and theoretical framework of the Kepler's problem, shows an equivalence between the computation of switching instants of the PWM and the eccentric anomaly of the planet orbit around the sun, giving a simple stability criterion and a sufficient condition for the absence of solutions of the harmonic balance equation and, therefore, the probable absence of limit cycles of period multiple of that characteristic of the modulator. The derived stability criterion, by using the describing function method, is successively compared with the local stability of the closed-loop PWM system for first and second order plants. In the first case it has been formally proved that the proposed criterion ensures the local stability of an equilibrium point, while in the second one a Monte-Carlo simulation has confirmed that the selection of the modulator parameters, according to the proposed criterion, gives an effective method to avoid limit cycles and to ensure the local stability.
\end{abstract}

Keywords Pulse width modulation · describing function $\cdot$ Kapteyn series $\cdot$ Bessel functions

\section{Introduction}

Pulse width modulated (PWM) switching converters are widely used in industry and in consumer products and an extensive literature exists on this topic (see [1] and the references therein). PWM systems constitute a class of systems with discontinuous controls in which the control actions are determined on the basis of the error signal: in each sampling period, characteristic of the modulator, the control signal is enabled only for a fraction of time proportional to the error magnitude. The main advantages of this particular control mode are related to the power amplifier efficiency, since it essentially operates as an on-off device, and to the low cost for the engineering realization. Its relay-type electronic switching leads to amplifiers with reduced size, weight

Dipartimento di Elettronica Informatica e Sistemistica, Università degli Studi della Calabria, 87036, Rende (Cs), Italy 
and power dissipation [2]. However, the analysis of the PWM feedback control system can be extremely difficult, since the modulating device creates an effect equivalent to a non-linear non-instantaneous block [3]. The discontinuity control analysis can be approached by working with an approximate model obtained by the state-space averaging method which leads to a greater understanding of the dynamic performance of PWM converters although it is valid only for high frequency switching $[4,5]$. In the relevant literature, local and global stability problems have been considered for PWM feedback control systems with different regulators [6]. On the other hand, the describing function method has widely been used to determine the limit cycle and the dynamical behavior for the nonlinear systems [7-12]. As it is well known, finding conditions to predict and to eliminate limit cycles, the so-called ripple instability, for these control systems, is an important task. Many authors have detailed various approaches for the exact determination of limit cycles in PWM systems [13-16]. The use of describing function theory analysis of PWM systems without a reference input is well documented in [7]. The existing approaches substantially use the same logic: the shape of limit cycle mode, which defines the form of the nonlinearity output, is postulated; this nonlinearity output signal is processed through the linear part of the system to the nonlinearity input, finally, conditions which will produce the postulated output of the nonlinearity are sought. The system is then analyzed as a linear block interconnected with a nonlinear block described by its amplitude dependent transfer function. The resulting equations, for the amplitude and frequency, of presumed limit cycles are solved directly by a graphical procedure through the intersections of the frequency-response of the linear device and the critical locus (i.e, the inverse of the describing function) of the nonlinear ones in the Nyquist plane, otherwise by solving the nonlinear equations or a parameter optimization problem $[7,17,18]$. The advantage of the describing function method is that it can be viewed as a kind of harmonic balance method [5]. On the other hand, the fundamental limitation is that the form of the signal in input to the nonlinearity must be guessed in advance. Another difficulty is related to the possibility to have multiple solutions since the formulation of the problem, using the describing function, leads to a simultaneous set of nonlinear algebraic relations to be solved. Moreover, since the method is approximate, another limitation is that it is only able to indicate where probably a limit cycle can occur. Finally, the plant should be low-pass to attenuate higher harmonics that is to say the analysis with this method has to be cautious. Several types of describing functions have been derived. For instance, the case of multiple input nonlinearities was discussed in [7], while the case of random inputs describing functions can be found in $[8,19]$.

\subsection{The aim and the organization of the paper}

The aim of this paper is twofold. First, an interesting relation between the switching instants in the PWM modulator and the eccentric anomaly of the planet orbit around the sun is pointed out. Second, a sufficient condition to avoid limit cycles predicted by the dual-input describing function in a class of PWM feedback control systems is given. The method used herein reconsiders the dual-input describing function approach on the basis of an exact spectral analysis of the PWM output, and extends the results in [7] introducing a constant reference input. Moreover, in order to avoid probable limit cycles of period multiple of that characteristic of the modulator, a simple stability criterion is proposed. Finally, some simulation results, which confirm the validity of the proposed 
criterion, are shown. In particular, for a first order plant it will be formally proved that the describing function stability criterion ensures the local stability of an equilibrium point, while for a second order plant a Monte-Carlo simulation has confirmed that the selection of the modulator parameters, according to the proposed criterion, gives an effective method to avoid limit cycles and to ensure the local stability. The idea behind the proposed approach is that the implicit equation of the unknown PWM switching instants, when the input to the modulator is a biased sinusoidal signal due to the presence of a limit cycle, can be related to the Kepler's equation governing the trajectory of a planet around the sun [20]. The solution of the Kepler's problem allows to obtain the switching instants of the PWM output in terms of Kapteyn series as well as its Fourier coefficients and then to find an explicit expression of the describing function. The paper is organized as follows. Section 2 describes the main aspects of the Kepler's problem with a solution in terms of Kapteyn series; Sections 3 and 4 introduce the model of the PWM closed-loop system and an analysis for the local stability, respectively. In Section 5 an overview of dual-input describing function and main results are given. In order to corroborate the theoretical analysis, simulation results are reported in Section 6. Finally Section 7 is devoted to conclusions. Proofs of some results are reported in the Appendix.

\section{Kepler's equation}

Many problems in Celestial Mechanics require a solution to Kepler's equation. The Kepler transcendental equation

$$
E-\epsilon \sin (E)=M
$$

which links the eccentric anomaly of elliptic motion $E$, mean anomaly $M$ and eccentricity $\epsilon$, is of critical importance in gravitational mechanics. The basic physical meaning of this equation is better explained by Fig. 1 in which is depicted an ellipse, with eccentricity $\epsilon$, that is the orbit of a body moving about the stationary gravitating center placed in focus of the ellipse $S$. Denote by $C$ and $A$ the center and pericenter of the ellipse, respectively. Construct also a circle with its center at point $C$ and with a radius equal to the major semiaxis of the ellipse. At some time let the position of the rotating body be determined by point $P$. From $P$ we drop a perpendicular to the major axis of the ellipse and denote the foot of the perpendicular by letter $R$. Extend this perpendicular to intersect the circle at point $Q$. Then the angle $\angle A C Q$ is just the eccentric anomaly $E$. Suppose that the planet $P$, having passed through perihelion $A$, is at position $P$ after elapsed time $t$, it is possible to express the polar coordinates of $P,(r, v)$, relative to the sun in terms of $t$. Kepler's equation relates $E$ to time $t$ by means of a quantity

$$
M=\frac{2 \pi t}{\Xi},
$$

where $\Xi$ is the time required for the planet to complete one trip in its orbit around the sun. The quantity $M$ represents the average angular speed of the radius vector $S P$. Classical formulas to find $r$ and $v$ are

$$
r=a(1-\epsilon \cos (E))
$$


and

$$
\tan \left(\frac{v}{2}\right)=\sqrt{\frac{1+\epsilon}{1-\epsilon}} \tan \left(\frac{E}{2}\right)
$$

Therefore, if $\Xi$ and $M$ are known, it is possible to solve the Kepler's equation for $E$ and to determine position $(r, v)$ at time $t$ by using eqs. (3) and (4).

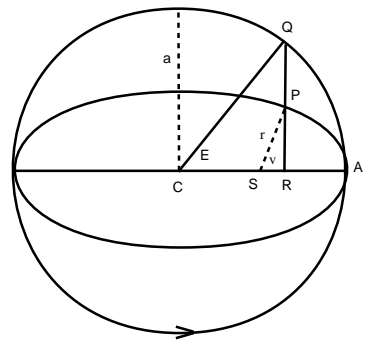

Fig. 1 Graphical representation of orbital elements.

Although there exists a unique solution of the Kepler's problem, there are many methods to describe or approximate it [21-24]. The history of the search for exact and approximate solutions of the Kepler's equation, along with the analytical and numerical methods used for this purpose, is well covered in [20]. A solution in terms of Bessel functions of the first kind:

$$
J_{n}(x)=\frac{1}{\pi} \int_{0}^{\pi} \cos (x \sin (\theta)-n \theta) d \theta,
$$

was investigated by Kapteyn in his memoir in 1893 [25], where he studied the possibility of representing functions by series of the form

$$
f(x)=\sum_{n=0}^{\infty} h_{n}(f) J_{n}(n x),
$$

where $h_{n}(f)$ are coefficients depending on the function $f(x)$. Series in Eq. (6) are now called Kapteyn series.

Let $E=g(M)$ be the solution of the Kepler's equation and express the quantity $g(M)-M$ in the Fourier sine-series

$$
g(M)-M=\epsilon \sin (E)=\sum_{n=1}^{\infty} A_{n} \sin (n M),
$$


where

$$
\begin{aligned}
A_{n} & =\frac{2}{\pi} \int_{0}^{\pi}[g(M)-M] \sin (n M) d M \\
& =-\frac{2}{n \pi} \int_{0}^{\pi}[g(M)-M] d(\cos (n M)) \\
& =-\frac{2}{n \pi}\{[g(M)-M] \cos (n M)\}_{0}^{\pi}+\frac{2}{n \pi} \int_{0}^{\pi} \cos (n M)\left[g^{\prime}(M)-1\right] d M \\
& =\frac{2}{n \pi} \int_{0}^{\pi} \cos (n M) g^{\prime}(M) d M \\
& =\frac{2}{n \pi} \int_{0}^{\pi} \cos (n M) d(g(M)) .
\end{aligned}
$$

Since $M=E-\epsilon \sin (E)=g(M)-\epsilon \sin (g(M))$, then

$$
\begin{aligned}
A_{n} & =\frac{2}{n \pi} \int_{0}^{2 \pi} \cos [n g(M)-n \epsilon \sin (g(M))] d(g(M)) \\
& =\frac{2}{n}\left\{\frac{1}{\pi} \int_{0}^{\pi} \cos (n E-n \epsilon \sin (E)) d E\right\} \\
& =\frac{2}{n} J_{n}(n \epsilon) .
\end{aligned}
$$

Hence the solution of (1) in terms of Kapteyn series gives

$$
E=M+\sum_{n=1}^{\infty} \frac{2}{n} J_{n}(n \epsilon) \sin (n M) .
$$

The series on the right side rapidly converges when $\epsilon<1$, and it is still convergent when $\epsilon=1[26]$.

\section{System modeling}

The system which will be analyzed is depicted in Fig. 2. The plant is assumed to be linear, time-invariant with low-pass characteristic. The modulator output consists of a piecewise constant function in two subintervals, $\left[k T, k T+\tau_{k}\right)$ and $\left(k T+\tau_{k}, k T+T\right)$, where $\tau_{k} \in[0, T]$ represents the switching instant in the interval $[k T, k T+T)$ and $T$ is the constant sampling period.

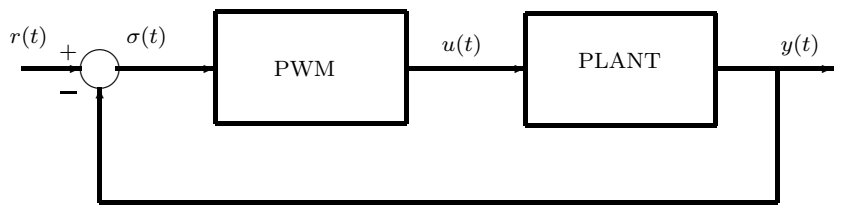

Fig. 2 PWM feedback control system. 
Fig. 3 shows the unipolar lead-type modulator output which is characterized by values equal to $+M$ or $-M$ in the first subinterval (according to the sign of the modulator input $\sigma(t)$ valued for $t=k T$ ) and equal to zero in the second subinterval:

$$
u(t)= \begin{cases}M \operatorname{sgn}[\sigma(k T)], & k T \leq t \leq k T+\tau_{k}, \\ 0, & k T+\tau_{k}<t<k T+T\end{cases}
$$

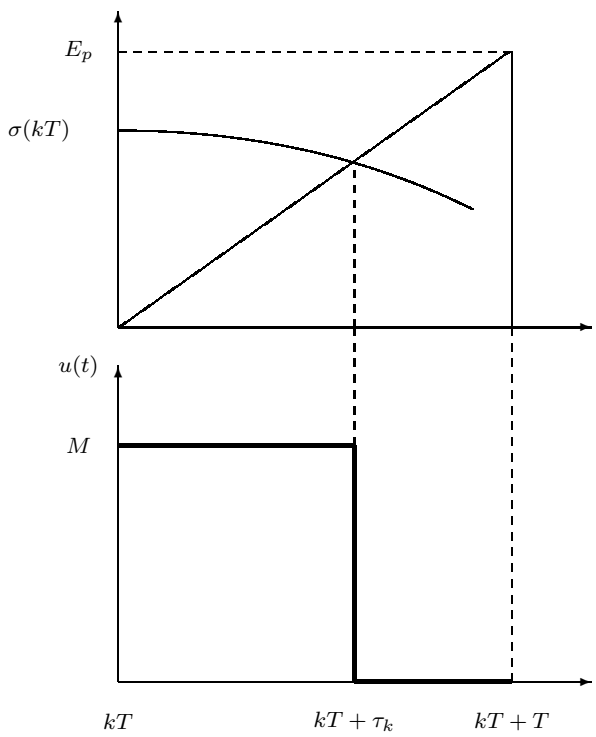

Fig. 3 Lead-type PWM modulator.

More general modulation strategies are described in [27].

As far as the evaluation of $\tau_{k}$ is concerned, its value is uniquely determined by a logic comparison between the input $\sigma(t)$ and a sawtooth carrier $c(t)$, of period $T$, which has the following expression:

$$
c(t)=\operatorname{sgn}[\sigma(k T)] \frac{E_{p}}{T}(t-k T), \quad k T \leq t \leq k T+T,
$$

where $E_{p}>0$ is the sawtooth amplitude. For the natural sampling modulator, the relationship enabling $\tau_{k}$ to be determined is:

$$
\sigma\left(k T+\tau_{k}\right)=\operatorname{sgn}[\sigma(k T)] E_{p} \frac{\tau_{k}}{T}
$$

where it is evident that $\tau_{k}$ is implicitly determined by the input behavior in the interval $\left[k T, k T+\tau_{k}\right.$ ), and it is assumed that there is a unique solution $\tau_{k}$ (see Remark 1 for details about this assumption). 


\section{Local stability and assumptions}

The equations describing the dynamical behavior of the control system are:

$$
\left\{\begin{array}{l}
\dot{x}(t)=A x(t)+b u(t), \\
y(t)=c^{T} x(t), \\
\sigma(t)=r-y(t)
\end{array}\right.
$$

where $x \in \mathbb{R}^{n}$ is the state, $y \in \mathbb{R}$ and $u \in \mathbb{R}$ (described by Eq. (11)) are respectively the output and the input of the plant, $r \in \mathbb{R}$ is the constant reference input, $A \in \mathbb{R}^{n \times n}$, $b, c \in \mathbb{R}^{n \times 1}$. Moreover it will be assumed that $A$ is a full-rank matrix.

Remark 1 It has been assumed that Eq. (13) has a unique solution $\tau_{k}$ belonging to $[0, T]$. Obviously, this hypothesis is not sustainable from a mathematical point of view, unless a set of input functions, which guarantees this hypothesis, is considered. However, such assumption does not represent a limitation if the sampling period is suitably chosen taking into account the time constants of the controlled process, in such a way to guarantee the uniqueness of the solution. In addition we can consider a set of input functions such that the unique solution of Eq. (13) belongs to $[0, T]$ (no modulation saturation), with the only implication that a small signal analysis of the control loop needs to be performed. In the implementation of the PWM modulator logic, the value of $\tau_{k}$ is assumed equal to $T$ if no solution exists in the interval $[k T, k T+T]$.

For $t \in\left[k T, k T+\tau_{k}\right]$ one has:

$$
x(t)=e^{A(t-k T)} x(k T)+\int_{k T}^{t} e^{A(t-\xi)} b u(\xi) d \xi .
$$

By substituting the expression of $u(t)$ given in Eq. (11) and by using standard algebraic manipulation, it follows:

$$
x(t)=e^{A(t-k T)} x(k T)+M \operatorname{sgn}[\sigma(k T)] A^{-1}\left[e^{A(t-k T)}-I\right] b,
$$

where $I$ is the identity matrix of dimension $n$.

In the second subinterval $t \in\left[k T+\tau_{k}, k T+T\right)$, the state system is described as:

$$
x(t)=e^{A\left(t-k T-\tau_{k}\right)} x\left(k T+\tau_{k}\right) .
$$

Therefore from Eq. (16) it is possible to get the state value in the switching instant $k T+\tau_{k}$ :

$$
x\left(k T+\tau_{k}\right)=e^{A \tau_{k}} x(k T)+M \operatorname{sgn}[\sigma(k T)] A^{-1}\left[e^{A \tau_{k}}-I\right] b,
$$

which can be substituted in Eq. (17):

$$
x(k T+T)=e^{A T} x(k T)+M \operatorname{sgn}[\sigma(k T)] A^{-1}\left[e^{A T}-e^{A\left(T-\tau_{k}\right)}\right] b .
$$

Since

$$
\sigma(t)=r-c^{T} x(t)
$$

then Eq. (13) can be rewritten as:

$$
c^{T} x\left(k T+\tau_{k}\right)+\frac{E_{p}}{T} \tau_{k} \operatorname{sgn}\left[\sigma\left(k T+\tau_{k}\right)\right]-r=0 .
$$


Note that, by the hypothesis of a unique switching instant in the interval $[k T, k T+$ $T)$, it must be that $\operatorname{sgn}\left[\sigma\left(k T+\tau_{k}\right)\right]=\operatorname{sgn}[\sigma(k T)]$, therefore Eq. (21) becomes:

$$
c^{T} e^{A \tau_{k}} x(k T)+M \operatorname{sgn}[\sigma(k T)] c^{T} A^{-1}\left[e^{A \tau_{k}}-I\right] b+\frac{E_{p}}{T} \tau_{k} \operatorname{sgn}[\sigma(k T)]-r=0 .
$$

Eqs. (19) and (22) describe the dynamic of the PWM feedback control system.

Let us suppose that an equilibrium point exists. This means that, after a transient, the state at the beginning of the period is equal to the state at the end of the period. Moreover, if $r$ is a constant reference input, then the value of $\tau_{k}$, the value of the error function at the beginning of the period, i.e. $\sigma(k T)$, and consequently $\operatorname{sgn}[\sigma(k T)]$, must be constant for each period $k$. Indicating with $\left(x_{\infty}, \tau_{\infty}\right)$ the equilibrium point, it will be

$$
\begin{aligned}
& x(k T+T)=x(k T)=x_{\infty}, \\
& \tau_{k}=\tau_{\infty}, \\
& \operatorname{sgn}[\sigma(k T)]=\operatorname{sgn}_{\infty} .
\end{aligned} \forall k
$$

Eqs. (19) and (22) with (23) become respectively

$$
x_{\infty}=M \operatorname{sgn}_{\infty} A^{-1}\left[I-e^{A T}\right]^{-1}\left(e^{A T}-e^{A\left(T-\tau_{\infty}\right)}\right) b
$$

and

$$
M c^{T} A^{-1}\left[I-e^{A T}\right]^{-1}\left(e^{A \tau_{\infty}}-I\right) b+\frac{E_{p}}{T} \tau_{\infty}-r \operatorname{sgn}_{\infty}=0 .
$$

Remark 2 Note that solutions of Eqs. (24) and (25) give formal conditions to the existence of equilibrium points. In [6] sufficient conditions for the uniform asymptotic stability in the large of the trivial solution $x_{\infty}=0(r=0)$ are investigated.

In order to obtain the necessary and sufficient condition for local stability, we consider the linearized discrete model in the neighborhood of the equilibrium point $\left(x_{\infty}, \tau_{\infty}\right)[28]:$

$$
\left\{\begin{array}{l}
d x(k T+T)=F d x(k T), \\
F=e^{A T}-\frac{1}{L} e^{A T} b c^{T},
\end{array}\right.
$$

where

$$
L=c^{T}\left[I-e^{A T}\right]^{-1}\left(e^{A \tau_{\infty}}-e^{A T}\right) b+\frac{E_{p}}{T M} .
$$

For the sake of the reader, Eqs. (26) and (27) are derived in the Appendix A.

Hence, the equilibrium point is locally asymptotically stable if and only if the eigenvalues of the matrix $F$ belong to the unit circle. Therefore, by Eqs. (26)-(27), it is possible to obtain the critical value of $E_{p}$ as a function of $T$ and $\tau_{\infty} \in[0, T]$. Note however that our interest is in a value of $E_{p}$ which ensures the local stability, for all values of $\tau_{k}$ in $[0, T]$. Such value will be compared with that one given by the dual-input describing function method, which is valid for all values of $\tau_{k}$ in $[0, T]$ and for every constant reference input $r$. 


\section{Overview of dual-input describing function and main results}

Suppose that a limit cycle takes place in the PWM closed-loop depicted in Fig. 2. On the basis of the well known peculiarities of the subharmonic modulation technique applied to the inverters, the PWM input $\sigma(t)$ and output $u(t)$ are periodic of period $N T$ with $N$ positive integer $[7,29]$. Therefore the switching process generates a sinusoidal input to the PWM modulator of amplitude $\mu E_{p}$ and frequency $\omega_{c}=\frac{2 \pi}{N T}$ with a DC offset $\mu_{0} E_{p}$ :

$$
\sigma(t)=\mu_{0} E_{p}+\mu E_{p} \sin \left(\omega_{c} t+\phi\right) .
$$

In Eq. (28) we consider $\mu<\mu_{0}<1-\mu$ so that the modulation saturation is avoided.

Remark 3 We assume that the system is operating in a condition of limit cycle in the neighborhood of an equilibrium point and that such oscillation does not involve a change of sign in the logic modulator. Therefore $\operatorname{sgn}[\sigma(k T)]$ is assumed equal to 1 in the sequel. Although such assumption should seem restrictive, however, as it will be evident in the simulation results, it does not affect the prediction capability of the method. Moreover it allows to simplify the expression of the describing function of the PWM modulator, giving the possibility of further theoretical investigations.

By using Fourier series, this periodic function $u(t)$ can be expanded as

$$
u(t)=b_{0}+\sum_{n=1}^{\infty}\left[b_{n} \cos \left(n \omega_{c} t\right)+a_{n} \sin \left(n \omega_{c} t\right)\right]
$$

where the Fourier coefficients are determined by

$$
\begin{aligned}
& b_{0}=\frac{1}{N T} \int_{0}^{N T} u(t) d t, \quad b_{n}=\frac{2}{N T} \int_{0}^{N T} u(t) \cos \left(n \omega_{c} t\right) d t, \\
& a_{n}=\frac{2}{N T} \int_{0}^{N T} u(t) \sin \left(n \omega_{c} t\right) d t .
\end{aligned}
$$

The fundamental component of $u(t)$ is described by

$$
u(t) \approx b_{0}+b_{1} \cos \left(\omega_{c} t\right)+a_{1} \sin \left(\omega_{c} t\right)=b_{0}+U_{1} \sin \left(\omega_{c} t+\phi_{1}\right)
$$

where $U_{1}=\sqrt{a_{1}^{2}+b_{1}^{2}}$ and $\phi_{1}=\tan ^{-1}\left(b_{1} / a_{1}\right)$.

The dual-input describing function of the nonlinear element is the complex ratio between the fundamental component of the non linear elements and the input [30], i.e.

$$
\left\{\begin{array}{l}
D_{0}=\frac{b_{0}}{\mu_{0} E_{p}}, \\
D_{1}=\frac{U_{1} e^{j \phi_{1}}}{\mu E_{p} e^{j \phi}}=\frac{a_{1}+j b_{1}}{\mu E_{p} e^{j \phi}} .
\end{array}\right.
$$

Eq. (13), particularized to the input $\sigma(t)$ of Eq. (28), gives the following expression of the switching instant $\tau_{k}$ in the generic interval $[k T, k T+T)$ :

$$
\frac{\tau_{k}}{T}=\mu_{0}+\mu \sin \left(\frac{2 \pi}{N} k+\frac{2 \pi}{N} \frac{\tau_{k}}{T}+\phi\right) .
$$

Eq. (33) can be rewritten as:

$$
E_{k}-\epsilon \sin \left(E_{k}\right)=M_{k}
$$


where

$$
\begin{gathered}
E_{k}=\frac{2 \pi}{N} k+\frac{2 \pi}{N} \frac{\tau_{k}}{T}+\phi, \\
\epsilon=\frac{2 \pi}{N} \mu
\end{gathered}
$$

and

$$
M_{k}=\frac{2 \pi}{N} \mu_{0}+\frac{2 \pi}{N} k+\phi
$$

Remark 4 From Eq. (36) it is necessary that $\mu \leq \min \left\{1, \frac{N}{2 \pi}\right\}$ to guarantee $\epsilon \leq 1$. Such an assumption does not affect the validity of the proposed approach because, as it will be clear afterwards, the sufficient conditions, provided by the method, refer to the onset of the limit cycle, i.e. $\mu \approx 0$.

Remark 5 It is important to observe that Eq. (34) is related to the problem of finding the abscissa corresponding to the intersection of the function $\sin \left(E_{k}\right)$ with the line $\frac{E_{k}-M_{k}}{\epsilon_{k}}$ (see Fig. 4). Therefore, any given problem conforming this geometrical structure, can be expressed through an expansion in terms of Kapteyn series.

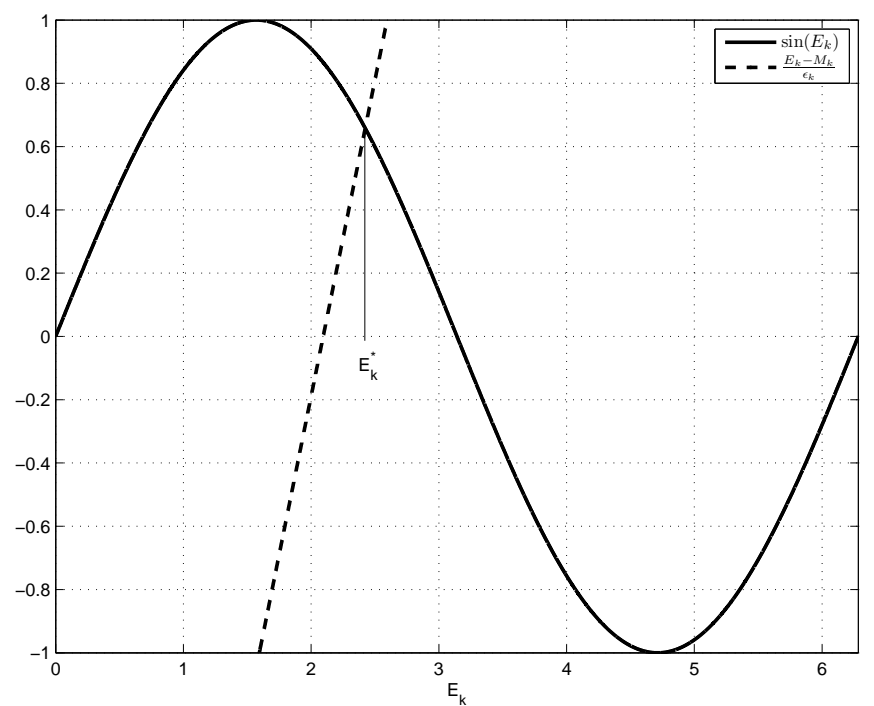

Fig. 4 Graphic solution of the Kepler's equation.

Eq. (34) is formally equivalent to the Kepler's equation (1). Hence the solution of (34) in terms of Kapteyn series gives:

$$
E_{k}=M_{k}+2 \sum_{n=1}^{\infty} \frac{1}{n} J_{n}(n \epsilon) \sin \left(n M_{k}\right) .
$$


From Eq. (38), an explicit relation of $\tau_{k} / T$ in terms of Kapteyn series can be obtained:

$$
\frac{\tau_{k}}{T}=\mu_{0}+\frac{N}{\pi} \sum_{n=1}^{\infty} \frac{1}{n} J_{n}(n \epsilon) \sin \left(n M_{k}\right) .
$$

Taking into account the expression of $u(t)$ which can be compactly rewritten as

$$
u(t)=M \sum_{k=0}^{N-1}\left(\delta_{-1}(t-k T)-\delta_{-1}\left(t-k T-\tau_{k}\right)\right),
$$

with $\delta_{-1}(t)=\left\{\begin{array}{l}1, t \geq 0, \\ 0, \text { otherwise }\end{array}\right.$, the mean value $b_{0}$ and the first coefficients $b_{1}$ and $a_{1}$ of $u(t)$ become:

$$
\begin{gathered}
b_{0}=\frac{M}{N} \sum_{k=0}^{N-1} \frac{\tau_{k}}{T}=M \mu_{0}+\frac{M}{\pi} \sum_{p=1}^{\infty} \frac{1}{p} J_{p N}(p N \epsilon) \sin \left[p\left(N \phi+2 \mu_{0} \pi\right)\right], \\
b_{1}=\frac{2 M}{N T} \sum_{k=0}^{N-1} \int_{k T}^{k T+\tau_{k}} \cos \left(\frac{2 \pi}{N T} t\right) d t=\frac{2 M}{\pi} \sum_{k=0}^{N-1} \sin \left(\frac{\pi \tau_{k}}{N T}\right) \cos \left(\frac{\pi}{N T}\left(2 k T+\tau_{k}\right)\right), \\
a_{1}=\frac{2 M}{N T} \sum_{k=0}^{N-1} \int_{k T}^{k T+\tau_{k}} \sin \left(\frac{2 \pi}{N T} t\right) d t=\frac{2 M}{\pi} \sum_{k=0}^{N-1} \sin \left(\frac{\pi \tau_{k}}{N T}\right) \sin \left(\frac{\pi}{N T}\left(2 k T+\tau_{k}\right)\right) .
\end{gathered}
$$

Let us consider the complex variable $c_{1}=a_{1}+j b_{1}$ which can be rewritten as:

$$
c_{1}=-\frac{M}{\pi} e^{j \phi} \sum_{k=0}^{N-1}\left[\cos \left(E_{k}\right)-j \sin \left(E_{k}\right)\right]
$$

Considering the following expressions associated with the Kepler-Bessel expansions [26]:

$$
\begin{gathered}
\cos \left(E_{k}\right)=-\frac{\epsilon}{2}+\sum_{n=1}^{\infty} \frac{1}{n}\left[J_{n-1}(n \epsilon)-J_{n+1}(n \epsilon)\right] \cos \left(n M_{k}\right), \\
\sin \left(E_{k}\right)=\sum_{n=1}^{\infty} \frac{1}{n}\left[J_{n-1}(n \epsilon)+J_{n+1}(n \epsilon)\right] \sin \left(n M_{k}\right),
\end{gathered}
$$

the Eq. (43) becomes:

$c_{1}=-\frac{N \epsilon}{2}+\sum_{k=0}^{N-1} \sum_{n=1}^{\infty}\left\{\frac{1}{n}\left[J_{n-1}(n \epsilon)-J_{n+1}(n \epsilon)\right] \cos \left(n M_{k}\right)-j\left[J_{n-1}(n \epsilon)+J_{n+1}(n \epsilon)\right] \sin \left(n M_{k}\right)\right\}$.

By using the following relations, (see Lemma 1 in Appendix 7 for a sketch of proof):

$$
\begin{aligned}
& \sum_{k=0}^{N-1} \cos \left(n M_{k}\right)=N \cos \left(p\left(N \phi+2 \mu_{0} \pi\right)\right), n=p N, p \in \mathbb{N}, \\
& \sum_{k=0}^{N-1} \sin \left(n M_{k}\right)=N \sin \left(p\left(N \phi+2 \mu_{0} \pi\right)\right), n=p N, p \in \mathbb{N},
\end{aligned}
$$


the Eq. (46) is simplified as:

$c_{1}=\frac{M}{\pi} e^{j \phi}\left\{\frac{N}{2} \epsilon+\sum_{p=1}^{\infty} \frac{1}{p} J_{p N+1}(p N \epsilon) e^{j p\left(2 \mu_{0} \pi+N \phi\right)}-\sum_{p=1}^{\infty} \frac{1}{p} J_{p N-1}(p N \epsilon) e^{-j p\left(2 \mu_{0} \pi+N \phi\right)}\right\}$.

Finally, by using Eq. (48) and the property of Bessel functions [26]

$$
\frac{J_{n}(z)}{z}=\frac{1}{2 n}\left(J_{n-1}(z)+J_{n+1}(z)\right),
$$

an expression of $D_{1}$ is obtained:

$$
\begin{aligned}
& D_{1}=\frac{2 M}{E_{p}}\left\{\frac{1}{2}+\sum_{p=1}^{\infty} \frac{1}{2 p N+2}\left(J_{p N}(2 \pi \mu p)+J_{p N+2}(2 \pi \mu p)\right) e^{j p\left(2 \mu_{0} \pi+N \phi\right)}+\right. \\
& \left.-\sum_{p=1}^{\infty} \frac{1}{2 p N-2}\left(J_{p N-2}(2 \pi \mu p)+J_{p N}(2 \pi \mu p)\right) e^{-j p\left(2 \mu_{0} \pi+N \phi\right)}\right\} .
\end{aligned}
$$

The next theorem gives a sufficient condition for the ripple stability of a PWM closed-loop control system.

Theorem 1 A sufficient condition which guarantees the absence of solution of the harmonic balance equation and, therefore, the absence of oscillations, predicted by the dual-input describing function, of period $N T$ with $N \geq 2$, for the PWM feedback control system, is

$$
\left|G\left(j \frac{\pi}{T}\right)\right|<\frac{E_{p}}{2 M} .
$$

Proof Let us consider the auxiliary variables $\alpha=2 \pi \mu$ and $\beta=2 \mu_{0} \pi+N \phi$. The function $D_{1}$ can be rewritten as

$$
D_{1}=u(\alpha, \beta)+j v(\alpha, \beta),
$$

where

$u(\alpha, \beta)=\frac{2 M}{E_{p}}\left\{\frac{1}{2}+\sum_{p=1}^{\infty} \cos (p \beta)\left[\frac{J_{p N}(p \alpha)+J_{p N+2}(p \alpha)}{2 p N+2}-\frac{J_{p N-2}(p \alpha)+J_{p N}(p \alpha)}{2 p N-2}\right]\right\}$,

and

$$
v(\alpha, \beta)=\frac{2 M}{E_{p}}\left\{\sum_{p=1}^{\infty} \sin (p \beta)\left[\frac{J_{p N}(p \alpha)+J_{p N+2}(p \alpha)}{2 p N+2}+\frac{J_{p N-2}(p \alpha)+J_{p N}(p \alpha)}{2 p N-2}\right]\right\} .
$$

If $\left|D_{1}\right|_{\max }$ denotes the maximum absolute value of $D_{1}$, then a sufficient condition for the PWM feedback control system to be free of oscillations with period $N T$ is

$$
\left|G\left(j \frac{2 \pi}{N T}\right)\right|<\frac{1}{\left|D_{1}\right| \max } .
$$

Since $\left|D_{1}\right|=\sqrt{u(\alpha, \beta)^{2}+v(\alpha, \beta)^{2}}$, then the values of $\alpha$ and $\beta$ which maximize $\left|D_{1}\right|$ are obtained by seeking the values that satisfy the following two equations:

$$
u \frac{\partial u(\alpha, \beta)}{\partial \alpha}+v \frac{\partial v(\alpha, \beta)}{\partial \alpha}=0
$$




$$
u \frac{\partial u(\alpha, \beta)}{\partial \beta}+v \frac{\partial v(\alpha, \beta)}{\partial \beta}=0 .
$$

By simple computations, the following non trivial solutions are obtained:

$$
\left\{\begin{array}{c}
N=2:(\alpha, \beta)=(0, \pi), \quad H=\frac{M}{E_{p}}\left(\begin{array}{cc}
-\frac{1}{3} & 0 \\
0 & -\frac{1}{2}
\end{array}\right), \quad\left|D_{1}\right|=\frac{2 M}{E_{p}} \\
N=3:(\alpha, \beta)_{1}=\left(0, \frac{\pi}{2}\right), \quad H=\frac{M}{E_{p}}\left(\begin{array}{cc}
\frac{1}{16} & \frac{1}{4} \\
\frac{1}{4} & 0
\end{array}\right) \\
(\alpha, \beta)_{2}=\left(0, \frac{3 \pi}{2}\right), H=\frac{M}{E_{p}}\left(\begin{array}{cc}
\frac{1}{16} & -\frac{1}{4} \\
-\frac{1}{4} & 0
\end{array}\right) \\
N=4:(\alpha, \beta)=(0,-), \quad H=\frac{M}{E_{p}}\left(\begin{array}{cc}
-\frac{\cos (\beta)}{12} & 0 \\
0 & 0
\end{array}\right) \\
N \geq 5:(\alpha, \beta)=(0,-), \quad H=\left(\begin{array}{ll}
0 & 0 \\
0 & 0
\end{array}\right) .
\end{array}\right.
$$

In Eq. (56), $H$ is the Hessian matrix obtained for each stationary point after standard but tedious manipulations by using the property of the Bessel functions [26]

$$
\frac{\partial J_{n}(p \alpha)}{\partial \alpha}=\frac{p}{2}\left[J_{n-1}(p \alpha)-J_{n+1}(p \alpha)\right]
$$

and by evaluating the second-order partial derivatives of $\left|D_{1}\right|$ respect to $\alpha$ and $\beta$ in the stationary point.

On the basis of the above considerations, since the unique maximum of $\left|D_{1}\right|$ is for $N=2$, then one can state that the following condition

$$
\left|G\left(j \frac{\pi}{T}\right)\right|<\frac{E_{p}}{2 M}
$$

is sufficient to ensure the absence of limit cycles of period $N T, N \geq 2$ for the considered control system.

Remark 6 Note that the expression of $D_{0}$ in Eq. (32) for $N=2, \alpha=0$ and $\beta=\pi$ gives the value of $\mu_{0}$ :

$$
\mu_{0}=\frac{r}{E_{p}+G(0) M}
$$

where $G(0)=-c^{T} A^{-1} b$ is the static gain of the process.

\section{Simulation results}

In this section the effectiveness of the proposed stability criterion is investigated through comparisons with local stability conditions. In the following, without loss of generality, we consider $M=1$; moreover the process time constants will be normalized in the interval $[0,1]$. 
6.1 Example 1.

The describing function is tested for a first order controlled plant

$$
G(s)=\frac{1}{s \gamma+1} .
$$

The inequality in Eq. (51), solved for $E_{p}$, gives the critical value of $E_{p}$, namely $E_{p_{d f}}$ :

$$
E_{p_{d f}}=\frac{2}{\sqrt{\pi^{2} \frac{\gamma^{2}}{T^{2}}+1}} .
$$

By Eqs. (26) and (27) the expressions of $N$ and $F$ are respectively

$$
\begin{gathered}
L=\frac{1-e^{\frac{T-\tau_{\infty}}{\gamma}}}{\gamma\left(1-e^{\frac{T}{\gamma}}\right)}+\frac{E_{p}}{T}, \\
F=e^{-\frac{T}{\gamma}}\left(1-\frac{1}{\frac{1-e^{\frac{T-\tau_{\infty}}{\gamma}}}{1-e^{\frac{T}{\gamma}}}+\frac{E_{p} \gamma}{T}}\right) .
\end{gathered}
$$

Note that the derivative of $F$ respect to $\tau_{\infty}$ :

$$
\frac{\partial F}{\partial \tau_{\infty}}=-\frac{T^{2}}{\gamma} \frac{e^{\frac{\tau_{\infty}}{\gamma}}\left(e^{\frac{T}{\gamma}}-1\right)}{\left(E_{p} \gamma e^{\frac{T+\tau_{\infty}}{\gamma}}+T e^{\frac{T}{\gamma}}-\left(E_{p} \gamma+T\right) e^{\frac{\tau_{\infty}}{\gamma}}\right)^{2}}
$$

is less than zero for each $\tau_{\infty} \in[0, T]$.

Therefore the equilibrium point is locally stable if and only if the two following inequality are satisfied:

$$
\begin{gathered}
\lim _{\tau_{\infty} \rightarrow 0} F=\frac{E_{p} \gamma}{E_{p} \gamma+T} e^{-\frac{T}{\gamma}}<1, \\
\lim _{\tau_{\infty} \rightarrow T} F=\frac{E_{p} \gamma-T}{E_{p} \gamma} e^{-\frac{T}{\gamma}}>-1 .
\end{gathered}
$$

Since Eq. (63) is satisfied $\forall \gamma>0$, then from Eq. (64) the critical value of $E_{p}$, namely $E_{p_{l s}}$ which assures the local stability of the equilibrium point is:

$$
E_{p_{l s}}=\frac{T}{\gamma\left(1+e^{\frac{T}{\gamma}}\right)} .
$$

The ratio between $E_{p_{l s}}$ and $E_{p_{d f}}$ can be rewritten as:

$$
\frac{E_{p_{l s}}}{E_{p_{d f}}}=\frac{x \sqrt{1+\frac{\pi^{2}}{x^{2}}}}{2\left(1+e^{x}\right)}
$$

with $x=\frac{T}{\gamma}$, and it is easy to show that $E_{p_{l s}}<E_{p_{d f}}$ for all $x \geq 0$. Therefore the proposed criterion ensures the local stability of the equilibrium point for each ratio $\frac{\tau_{\infty}}{T} \in[0,1]$. 
6.2 Example 2.

A second order plant characterized by the following transfer function

$$
G(s)=\frac{s \xi_{3}+1}{\left(s \xi_{1}+1\right)\left(s \xi_{2}+1\right)}
$$

with $\xi_{1}, \xi_{2}, \xi_{3} \in[0,1]$ and $\xi_{1}>\xi_{2}$, is considered. The value of the modulator sampling period $T$ is randomly chosen in $\left[0, \min \left(\xi_{2}, \xi_{3}\right) / 2\right]$ in view of the fact that PWM systems are usually designed with a sampling period short compared with the response time of the linear part of the system, so the ripple at sampling frequency is well attenuated at the output of the system [7]. 10 Monte-Carlo simulations with $10^{3}$ plants have been conducted. In each simulation a value of $E_{p}=\rho E_{p_{d f}}$ with $\rho \in[0.1,1]$ was chosen and the number of trials in which the proposed criterion ensures the local stability is stored. Table 1 shows, for each simulation, the probability to have a local stable PWM closed-loop process, for each ratio $\frac{\tau_{\infty}}{T}$, when $E_{p}=\rho E_{p_{d f}}$. The obtained results show that the proposed criterion, even if approximate in nature due to the describing function approach, gives an effective method to avoid limit cycles and to ensure the local stability.

\begin{tabular}{|c||c|c|c|c|c|}
\hline$\rho$ & 0.1 & 0.2 & 0.3 & 0.4 & 0.5 \\
\hline Prob. & 0.5806 & 0.6325 & 0.7119 & 0.7764 & 0.8467 \\
\hline \hline$\rho$ & 0.6 & 0.7 & 0.8 & 0.9 & 1.0 \\
\hline Prob. & 0.9064 & 0.9652 & 0.9936 & 0.9997 & 1.0000 \\
\hline
\end{tabular}

Table 1 Probability to have a local stable process for different values of $\rho$.

\section{Concluding remarks}

The first contribution of this paper is represented by an expression of the dual-input describing function, in terms of Bessel functions, for a class of PWM modulator. The second important result is a sufficient condition to ensure the absence of limit cycles predicted by the dual-input describing function of period multiple of that characteristic of the modulator. All the results proposed are related to an equivalence between the switching instants of the modulator and the eccentricity of a planet orbit around the sun, described by Kepler's equation. Numerical simulations have shown the effectiveness of the condition in the case of a first and second order plant.

\section{References}

1. Gelig, A.Kh., Churilov, A.N.: Stability and Oscillations of Nonlinear Pulse-Modulated Systems, Birkhäuser, 1998.

2. Tsypkin, Y.Z., Churilov, A.N.: Relay Control Systems, Cambridge Univ. Press., 1984.

3. La Cava, M., Paletta, G., Picardi, C.: Stability analysis of PWM feedback control system with PID regulators, Int. Journal of Control, 39, 5, 987-1005, 1984.

4. Middlebrook, R.D., Cuk, S.: "A general unified approach to modeling switching-converter power stages, IEEE Power Electron. Specialists Conf. Record, 1976. 
5. Chung, S.C., Huang, S.R., Lin, C.I.: Applications of describing functions to estimate the continuous and discontinuous conduction mode for a DC-to-DC buck converter, IEEE. Proc. Electr. Power Appl., 147, 6, 513-519, 2000.

6. Hou, L., Michel, A.N.: Stability analysis of pulse-width-modulated feedback systems, Automatica, 37, 1335-1349, 2001.

7. Gelb, A., Vander Velde, W.E.: Multiple-input describing functions and nonlinear system design, McGraw-Hill, 1968.

8. Taylor, J.H.: Describing Functions, in Electrical Engineering Encyclopedia, John Wiley \& Sons, Inc., New York, 1999.

9. Kienitz, K.H.: On the Implementation of the Eigenvalue Method for Limit Cycle Determination in Nonlinear Systems, Nonlinear Dynamics, 45, 25-30, 2005.

10. Somieski, G.: An Eigenvalue Method for Calculation of Stability and Limit Cycles in Nonlinear Systems, Nonlinear Dynamics, 26, 3-22, 2001.

11. Sun, Y.J.: Existence and uniqueness of limit cycle for a class of nonlinear discrete-time systems, Chaos, Solitons \& Fractals, 38, 89-96, 2008

12. Zhang, T., Tadé, M.O., Tian, Y.C.: Linear estimate of the number of limit cycles for a class of non-linear systems, Chaos, Solitons \& Fractals, 31, 804-810, 2007.

13. Delfeld, F.R., Murphy, G.J.: Analysis of pulse-width-modulated control systems, IRE Trans. Aut. Control, 283-292, 1961.

14. Peterchev, A.V., Sanders, S.R.: Quantization resolution and limit cycling in digitally controlled PWM converters, IEEE Trans. Power Electron., 18, 301-308, 2003.

15. Abdelnour, G., Cheung, J., Chang, C., Tinetti, G.: Application of describing function in the transient response analysis of a three term fuzzy controller, IEEE Trans. Syst. Man Cybern., 23, 2, 607-610, 1993.

16. Heyns, L.J., Kruger, J.J.: Describing function based analysis of a nonlinear hydraulic transmission line, IEEE Trans. Contr. Syst. Technology, 2, 1, 31-35, 1994.

17. Soto, J.C., De La Sen, M.: Non-linear oscillations in nonperiodic sampling systems, Electronics Letters, 20, 20, 816-818, 1984.

18. Williamson, D.: Describing function analysis and oscillations in non-linear networks, Int. J. of Control, 24, 2, 283-296, 1976

19. Lim, Y.: Quasi-linear analysis of oscillating servo-systems with random inputs, IEEE Trans. Aut. Control, 10, 2, 164-171, 1965.

20. Colwell, P.: Solving Kepler's equation, Willmann-Bell, Inc., 1993.

21. Boyd, J.P.: Rootfinding for a transcendental equation without a first guess: Polynomialization of Kepler's equation through Chebyshev polynomial expansion of the sine, Appl. Numer. Math., 57, 12-18, 2007.

22. Dubinov, A.E., Galidakis, I.N.: Explicit Solution of the Kepler Equation, Physics of Particles and Nuclei Letters, 4, 3, 213-216, 2007.

23. Feinstein, S.A., McLaughlin, C.A.: Dynamic discretization method for solving Kepler's equation, Celestial Mech. Dyn. Astr., 96, 49-62, 2006.

24. Mortari, D., Clocchiatti, A.: Solving Kepler's Equation using Bézier curves, Celestial Mech. Dyn. Astr., 99, 45-57, 2007.

25. Kapteyn, M.W.: Recherches sur les fonctions de Fourier-Bessel, Ann. Sci. de l'Ecole Normale Superieure, 3, 10, 91-122, 1893.

26. Watson, G.N.: A treatise on the theory of the Bessel function, Cambridge Univ. Press., 1966.

27. Skoog, R.A., Blankenship, G.L.: Generalized pulse-modulated feedback systems: Norms, gains, lipschitz constants, and stability, IEEE Trans. Aut. Control, 15, 3, 300-315, 1970.

28. Eisinberg, A., Fedele, G., Frascino, D.: Local stability of a class of PWM feedback control systems, Tech. Rep., n. 1/08, Lab. Logistica, Univ. Calabria, Italy, 2008.

29. Balestrino, A., De Maria, G., Sciavicco, L.: On the ordinary and modified subharmonic control, II IFAC Symposium on control in power and electric drives, 155-163, 1977.

30. Mahalanabis, A.K., Nath, A.K.: On the dual-input describing function of a nonlinear element, IEEE Trans. Aut. Control, 10, 2, 203-204, 1965.

Appendix A

To obtain the linearized model on the equilibrium point $\left(x_{\infty}, \tau_{\infty}\right)$ (see Eqs. (26) and (27)) let us consider the first-term truncated Taylor series of the function $f\left(x(k T), \tau_{k}\right)$ :

$$
d f(k T+T)=\left.\frac{\partial f(k T+T)}{\partial x(k T)}\right|_{\left(x_{\infty}, \tau_{\infty}\right)} d x(k T)+\left.\frac{\partial f(k T+T)}{\partial \tau_{k}}\right|_{\left(x_{\infty}, \tau_{\infty}\right)} d \tau_{k} .
$$


Therefore, Eq. (26) becomes

$$
d x(k T+T)=e^{A T} d x(k T)+M \operatorname{sgn}_{\infty} e^{A\left(T-\tau_{\infty}\right)} b d \tau_{k} .
$$

Moreover, applying the implicit functions theorem to Eq. (27), one has

$$
c^{T} e^{A \tau_{\infty}} d x(k T)+\left[c^{T} A e^{A \tau_{\infty}} x_{\infty}+M \operatorname{sgn}_{\infty} c^{T} e^{A \tau_{\infty}} b+\frac{E_{p}}{T} \operatorname{sgn}_{\infty}\right] d \tau_{k}=0 .
$$

From Eq. (69), it is possible to find the value of $d \tau_{k}$ as function of $d x(k T)$ :

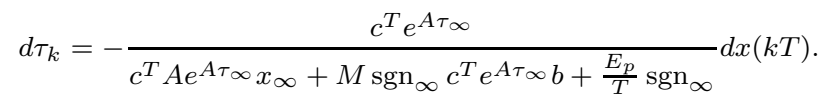

By substituting (70) in (68)

$$
d x(k T+T)=e^{A T} d x(k T)-\frac{e^{A T} b c^{T}}{\frac{c^{T} A e^{A \tau} x_{\infty}}{M \operatorname{sgn}_{\infty}}+c^{T} e^{A \tau_{\infty} b+\frac{E_{p}}{T M}}} d x(k T),
$$

and taking into account the expression of $x_{\infty}$, it follows that

$$
d x(k T+T)=e^{A T} d x(k T)+\frac{e^{A T} b c^{T}}{L} d x(k T),
$$

where

$$
L=c^{T}\left[I-e^{A T}\right]^{-1} e^{A T}\left(e^{A \tau_{\infty}}-I\right) b+c^{T} e^{A \tau_{\infty}} b+\frac{E_{p}}{T M} .
$$

Multiplying the second term of the previous expression by $\left[I-e^{A T}\right]^{-1}\left[I-e^{A T}\right]$

$$
L=c^{T}\left[I-e^{A T}\right]^{-1} e^{A T}\left(e^{A \tau_{\infty}}-I\right) b+c^{T}\left[I-e^{A T}\right]^{-1}\left[I-e^{A T}\right] e^{A \tau_{\infty} b}+\frac{E_{p}}{T M},
$$

it follows that

$$
L=c^{T}\left[I-e^{A T}\right]^{-1}\left(e^{A \tau_{\infty}}-e^{A T}\right) b+\frac{E_{p}}{T M} .
$$

\section{Lemma 1}

$$
\chi=\sum_{k=0}^{N-1} \sin \left(n M_{k}\right)=N \sin \left[p\left(N \phi+2 \mu_{0} \pi\right)\right], \quad n=p N, p \in \mathbb{N} .
$$

Proof Taking into account the expression of $M_{k}, \chi$ can be rewritten as:

$$
\chi=\cos \left[n\left(\frac{2 \pi}{N} \mu_{0}+\phi\right)\right] \sum_{k=0}^{N-1} \sin \left(\frac{2 \pi n}{N} k\right)+\sin \left[n\left(\frac{2 \pi}{N} \mu_{0}+\phi\right)\right] \sum_{k=0}^{N-1} \cos \left(\frac{2 \pi n}{N} k\right) .
$$

But

$$
\begin{gathered}
\sum_{k=0}^{N-1} \sin \left(\frac{2 \pi n}{N} k\right)=\frac{\sin (n \pi)}{\sin \left(\frac{n \pi}{N}\right)} \sin \left(\frac{n(N-1)}{N} \pi\right), \\
\sum_{k=0}^{N-1} \cos \left(\frac{2 \pi n}{N} k\right)=\sin (n \pi)\left(\cos (n \pi) \frac{\cos \left(\frac{n \pi}{N}\right)}{\sin \left(\frac{n \pi}{N}\right)}+\sin (n \pi)\right) .
\end{gathered}
$$

From the two previous equations, it follows that $n$ must be equal to $p N$ with $p \in \mathbb{N}$, then

$$
\frac{\sin (n \pi)}{\sin \left(\frac{n \pi}{N}\right)}=(-1)^{p(N-1)} N
$$


and

$$
\begin{gathered}
\sum_{k=0}^{N-1} \sin \left(\frac{2 \pi n}{N} k\right)=(-1)^{p(N-1)} N \sin [p(N-1) \pi]=0, \\
\sum_{k=0}^{N-1} \cos \left(\frac{2 \pi n}{N} k\right)=(-1)^{p(N-1)} N \cos (p N \pi) \cos (p \pi)=N,
\end{gathered}
$$

from which the proof follows.

With similar technicalities, the proof of the second summation in Eq. (47) can be approached. 